JOURNAL CLUB

\title{
Can continuous positive airway pressure reduce the incidence of cardiovascular disease in non-sleepy patients with obstructive sleep apnoea?
}

An association has been demonstrated between obstructive sleep apnoea (OSA) and both hypertension and cardiovascular events. Although observational studies suggest that treatment with continuous positive airways pressure (CPAP) can reduce blood pressure and the incidence of fatal and non-fatal cardiovascular events in patients with severe and moderate OSA, short term randomised controlled trials have failed to demonstrate a similar effect in non-sleepy patients.

The primary objective of this large, multicentre randomised controlled trial was to investigate the effect of CPAP on the incidence of hypertension and cardiovascular events in nonsleepy patients (Epworth Sleepiness Score of $\leq 10$, range 0-24) with OSA (apnoea-hypopnoea index of $20 \mathrm{~h}^{-1}$ or greater). The secondary objective was to assess an association between OSA severity and the incidence of hypertension or cardiovascular events.

Although a statistically significant reduction in the incidence of hypertension and cardiovascular events was not observed in the treatment group, there was a reduction in the incidence density ratio in a subgroup of patients with better adherence to treatment.

The study adds to the debate regarding potential cardiovascular benefits of CPAP in OSA and suggests that adequate adherence to treatment may result in a reduction in the incidence of hypertension or cardiovascular events in non-sleepy patients. Further larger studies with longer follow up should be conducted to confirm the results.

- Barbe F, Duran-Cantolla J, Sanchez-de-la-Torre, et al. Effect of continuous positive airway pressure on the incidence of hypertension and cardiovascular events in nonsleepy patients with obstructive sleep apnea. A randomized controlled trial. JAMA 2012;307:2161-8.

\begin{abstract}
Abiramy Jeyabalan
Correspondence to Dr Abiramy Jeyabalan, SpR North Bristol Lung Centre, Southmead Hospital, Westbury-on-Trym, Bristol BS10 5NB, UK; abiramyjeyabalan@hotmail.com
\end{abstract}

Competing interests None.

Provenance and peer review Not commissioned; internally peer reviewed.

To cite Jeyabalan A. Thorax 2013;68:686.

Published Online First 24 August 2012

Thorax 2013;68:686. doi:10.1136/thoraxjnl-2012-202515 